\title{
Editorial
}

\section{Recent Trends in Special Numbers and Special Functions and Polynomials}

\author{
Serkan Araci, ${ }^{1}$ Mehmet Acikgoz, ${ }^{2}$ Cenap Özel, ${ }^{3}$ H. M. Srivastava, ${ }^{4}$ and Toka Diagana ${ }^{5}$ \\ ${ }^{1}$ Department of Economics, Faculty of Economics, Administrative and Social Sciences, Hasan Kalyoncu University, \\ 27410 Gaziantep, Turkey \\ ${ }^{2}$ Department of Mathematics, Faculty of Arts and Sciences, University of Gaziantep, 27310 Gaziantep, Turkey \\ ${ }^{3}$ Department of Mathematics, Dokuz Eylül University, 35160 Izmir, Turkey \\ ${ }^{4}$ Department of Mathematics and Statistics, University of Victoria, Victoria, BC, Canada V8W 3R4 \\ ${ }^{5}$ Department of Mathematics, Howard University, 2441 6th, Street NW, Washington, DC 20059, USA \\ Correspondence should be addressed to Serkan Araci; mtsrkn@hotmail.com
}

Received 1 October 2015; Accepted 1 October 2015

Copyright (C) 2015 Serkan Araci et al. This is an open access article distributed under the Creative Commons Attribution License, which permits unrestricted use, distribution, and reproduction in any medium, provided the original work is properly cited.

Special numbers and polynomials play an extremely important role in the development of several branches of mathematics, physics, and engineering. They have many algebraic operations. Because of their finite evaluation schemes and closure under addition, multiplication, differentiation, integration, and composition, they are richly utilized in computational models of scientific and engineering problems. This issue contributes to the field of special functions and polynomials. An importance is placed on vital and important developments in classical analysis, number theory, mathematical analysis, mathematical physics, differential equations, and other parts of the natural sciences.

One of the aims of this special issue was to survey special numbers, special functions, and polynomials, where the essentiality of the certain class of analytic functions, generalized hypergeometric functions, Hurwitz-Lerch Zeta functions, Faber polynomial coefficients, the peak of noncentral Stirling numbers of the first kind, and structure between engineering mathematics are highlighted.

All manuscripts submitted to this special issue are subjected to a quick and closed peer-review process. The guest editors initially examined the manuscripts to check suitability of papers. Based on referees, who are well-known mathematicians, of this special issue, we got the best articles to be included in this issue. The results and properties of accepted papers are very interesting, well defined, and mathematically correct. The work is a relevant contribution to the fields of analysis and number theory.

Serkan Araci Mehmet Acikgoz

Cenap Özel

H. M. Srivastava

Toka Diagana 


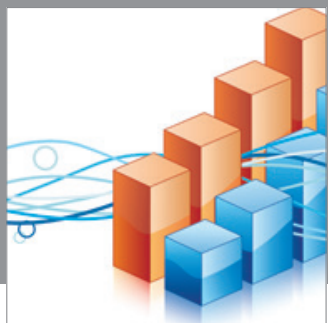

Advances in

Operations Research

mansans

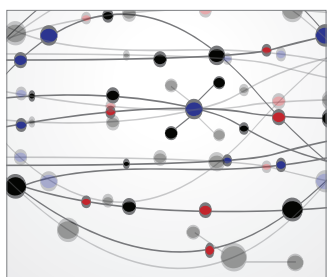

The Scientific World Journal
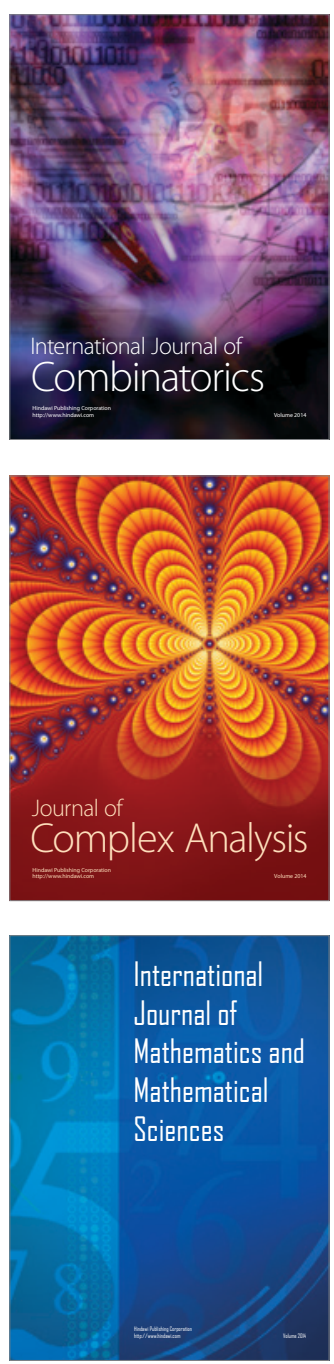
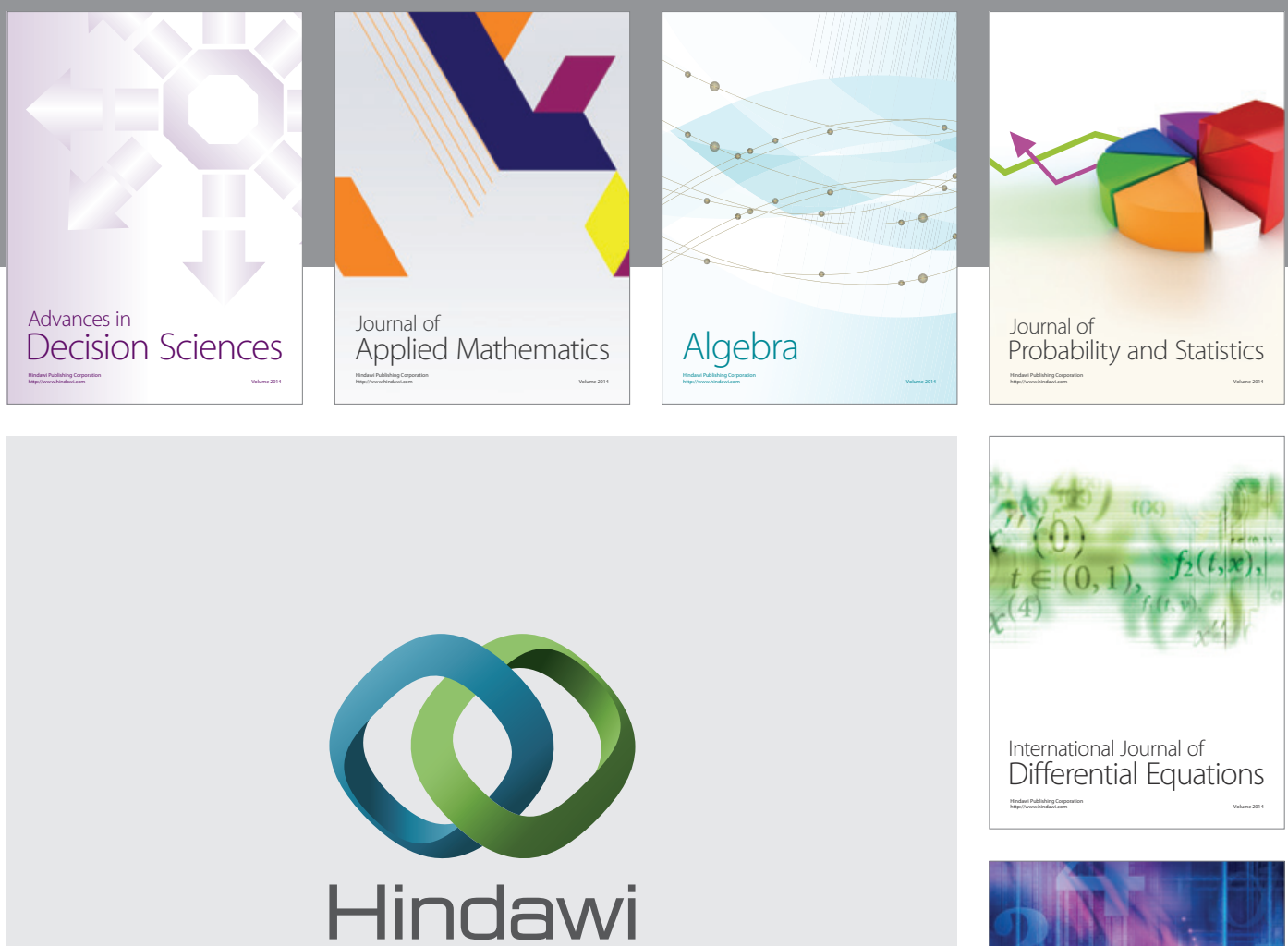

Submit your manuscripts at http://www.hindawi.com
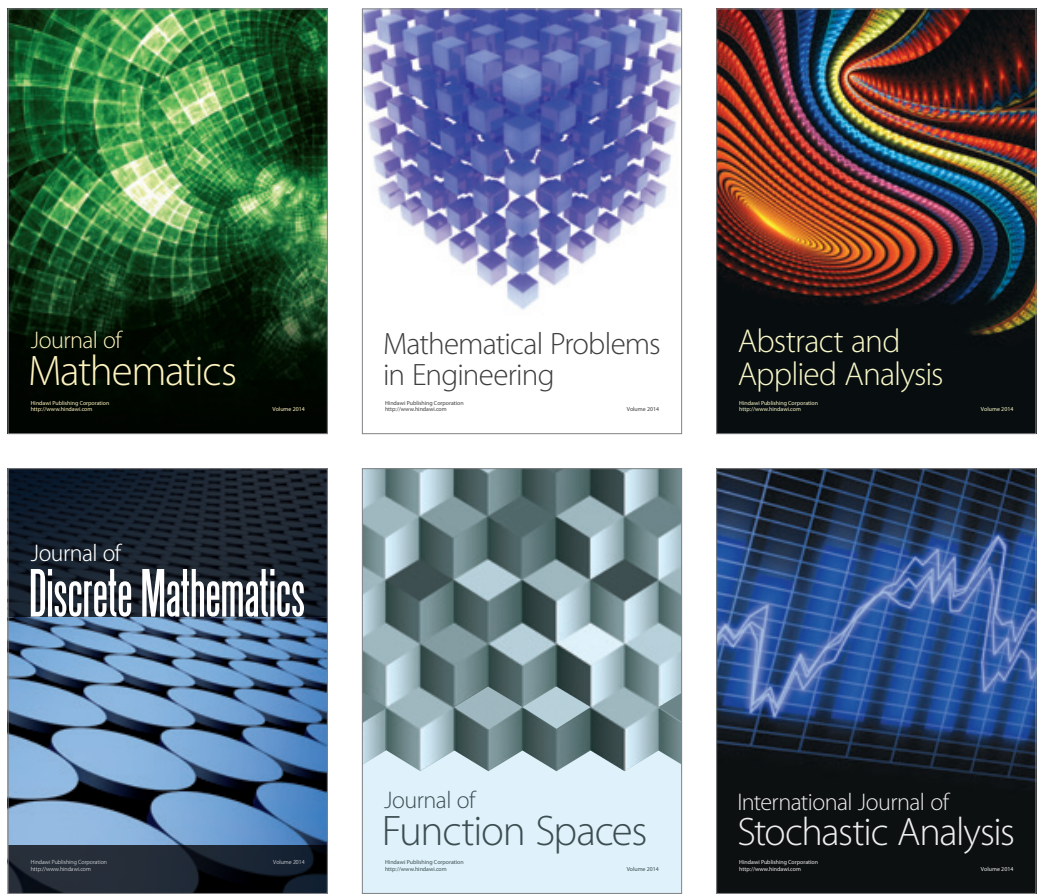

Journal of

Function Spaces

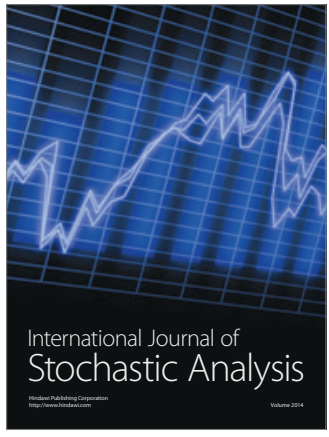

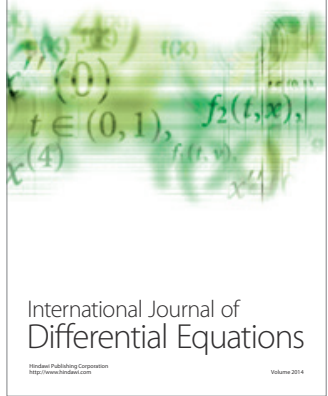
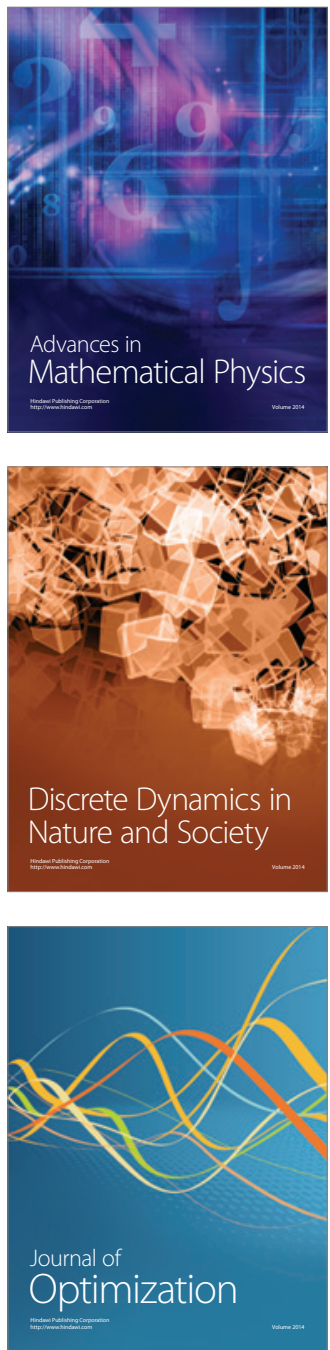